\title{
Emulador de Turbina de Viento Mediante el Control de Velocidad de un Motor de CD
}

\section{Wind Turbine Emulator Through DC Motor Speed Control}

\author{
O. A. Morfín-Garduño ${ }^{1}$, O. López-Hernández ${ }^{1}$, R. Acosta-Tejeda ${ }^{1}$
}

${ }^{1}$ Universidad Autónoma de Ciudad Juárez

\section{RESUMEN}

En el presente trabajo se diseña e implementa en tiempo real un controlador de velocidad del motor de corriente directa con excitación separada para emular la operación de una turbina eólica. Se define el modelo de la ecuación mecánica de la turbina eólica y del generador eléctrico, además del modelo del motor de corriente directa. Se aplica la técnica de linealización por retroalimentación de estados en combinación con la ley de control super-twisting de modos deslizantes de segundo orden en el diseño del controlador de velocidad en lazo cerrado, el cual sigue la velocidad de salida de un sistema eólico virtual que tiene como entrada un oscilador de viento, y con esto se logra emular la operación de la turbina. Se valida el emulador en tiempo real diseñando un observador asintótico del par de carga del motor de $\mathrm{CD}$, utilizando un grupo motor CD - dinamómetro y el kit DS1103 de dSPACE. Se valida el buen desempeño del emulador de turbina eólica en tiempo real con resultados gráficos del seguimiento de velocidad, de los márgenes de voltaje y corriente en el devanado de armadura, y de la estimación del par de carga con un observador asintótico.

PALABRAS CLAVE: Motor de CD; turbina de viento; algoritmo super-twisting.

\section{ABSTRACT}

The main goal of this research is to design and implement a real-time DC motor velocity controller in order to mimic the wind turbine operation. A model of the oscillation mechanical equation between the wind turbine and electrical generator, and a model of the separately excited DC motor are defined and simulated. The DC motor velocity controller that tracks the output velocity of the virtual wind system is designed from the DC motor model by applying the state feedback linearization technique. Then, the resulting controller mimics accurately the wind turbine. The super-twisting control technique of second order sliding modes is applied in the velocity controller design. The wind turbine emulator validation is performed in real-time using a motor DC-dynamometer group, and the DS1103 kit of dSPACE. An asymptotic observer to estimate the DC motor load torque, is required to perform the real-time test on the wind-turbine emulator. The emulator performance is validated in real time with the plot of the velocity tracking as control goal, the plot of the voltage supplied to armature winding as control input, the plot of the armature current, and the plot of load torque to shown the observer performance.

KEYWORDS: DC motor; wind turbine system; algorithm super-twisting.

Correspondencia:

DESTINATARIO: Onofre Amador Morfín-Garduño INSTITUCIÓN: Universidad Autónoma de Ciudad Juárez DIRECCIÓN: Avenida del Charro núm. 450 norte, col. Partido Romero, C. P. 32310, Ciudad Juárez, Chihuahua, México. CORREO ELECTRÓNICO: omorfin@uacj.mx

\section{Fecha de recepción:}

30 de junio de 2019

Fecha de aceptación:

10 de septiembre de 2019 


\section{INTRODUCCIÓN}

La generación de energía eléctrica basada en recursos fósiles está causando su reducción gradual por el amplio y continuo uso que se les da, además de que contaminan el ambiente con la emisión de gases de efecto invernadero. Surge entonces la necesidad de utilizar fuentes de energía renovables, con características necesarias para poder satisfacer la demanda creciente de electricidad, tales como la energía solar, del viento, de biomasa y geotérmica, entre otras. La energía eólica aventaja a la energía producida con recursos fósiles debido a que no genera agentes contaminantes para el ambiente y no reduce los recursos naturales del planeta. Sin embargo, el mayor reto con esta alternativa es la intermitencia en la energía generada, debido a las variaciones de velocidad del viento ${ }^{[1]}$.

El reto tecnológico en los sistemas eólicos consiste en maximizar la captura de energía del viento, realizar el proceso de conversión de energía de la forma más eficiente y cumplir con las normas de interconexión con el sistema eléctrico de potencia. Por ello, algunas investigaciones están enfocadas a controlar el generador de inducción y la interfaz de potencia entre el sistema eólico y la red eléctrica.

El diseño y construcción de la turbina se define mediante conceptos mecánicos y aerodinámicos y corresponde a otra área de estudio, además de que resulta muy costoso contar con la infraestructura de la turbina y un túnel de viento para investigaciones dirigidas al diseño de controladores del generador eléctrico y del inversor conectado a la red eléctrica. Por lo anteriormente expuesto, el objetivo del presente trabajo consiste en diseñar e implementar en tiempo real un emulador de turbina eólica que realice la mímica de su operación dinámica y, mediante un grupo de máquinas motor CD - generador CA, se pueda emular un sistema eólico para que, de este modo, las investigaciones se enfoquen exclusivamente al control del generador y su interconexión con la red eléctrica.

Los antecedentes principales de la presente investigación se presentan enseguida. Benaaouinte et al. ${ }^{[2]}$ presentan la emulación de una turbina eólica utilizando motores de imanes permanentes, donde se genera el torque y la velocidad necesarios para emular la operación de la turbina de viento; Guerrero et al. ${ }^{[3]}$ muestran el diseño de un estimador del par de carga que previene situaciones de sobre corrientes producidas por las perturbaciones externas en el sistema eólico; Sirouni et al. ${ }^{[4]}$, presentan una simulación de turbina eólica y motores de corriente directa utilizando los controladores PID y lógica difusa como técnicas de control, y así obtener las condiciones de operación ideales para un diseño de turbina de viento; y Morfin et al. ${ }^{[5]}$ muestran la simulación de un sistema eólico que comprende el modelo de una turbina eólica de 300 W que alimenta a un generador de inducción jaula de ardilla con una capacidad de $560 \mathrm{~W}$ (3/4 hp).

El sistema eólico tiene el objetivo de maximizar la captura de energía del viento a pesar de las variaciones en la velocidad de este definidas en un rango acotado. Por otra parte, en Voltolini et al. ${ }^{[6]}$ se presenta la emulación de la operación de una turbina eólica con el control de velocidad aplicado a un motor de inducción, así como el modelo del coeficiente de potencia para un tipo particular de diseño de turbina.

A partir de los modelos presentados en ${ }^{[5]}$ del motor de corriente directa y la turbina eólica en conjunto con el modelo de turbina planteado en ${ }^{[6]}$, se desarrolla en el presente trabajo la propuesta de emular el funcionamiento de la turbina eólica en el laboratorio mediante el control de velocidad en lazo cerrado del motor de corriente directa.

\section{METODOLOGÍA}

El emulador realiza la mímica de la operación de una turbina eólica, en donde un perfil de viento virtual incide en un sistema eólico virtual y tiene como variable de salida la velocidad en la flecha. Y es esta la velocidad de referencia para resolver el problema de seguimiento de trayectoria del controlador de velocidad del motor de corriente directa y cumplir con el objetivo de emular la operación de la turbina.

El emulador de turbina comprende: el exosistema que virtualmente define la velocidad de referencia; el control de velocidad en lazo cerrado del motor de corriente directa, en donde se aplica la técnica de linealización por retroalimentación de estados para el diseño de la variable de superficie; y el algoritmo super-twisting de modos deslizantes de segundo orden que fuerza hacia cero la superficie. En el cálculo de la superficie se utiliza la estimación del par de la carga mediante un observador asintótico de estados, y las corrientes del 
devanado de campo y de armadura, las cuales son monitoreadas mediante sensores de corriente. Se utiliza el software de visualización ControlDesk en el proceso de sintonización tanto del controlador como del observador de par.

En la Figura 1 se muestra la representación de la turbina de viento que es emulada por un motor de corriente directa.

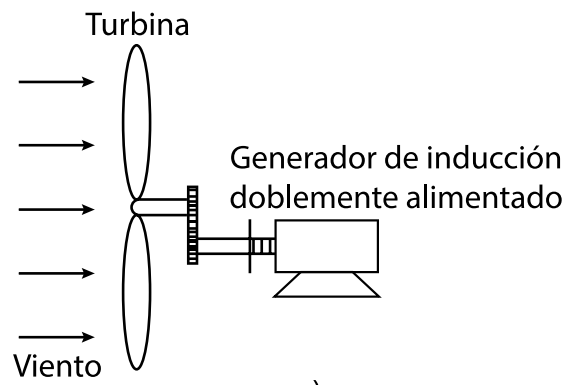

a)

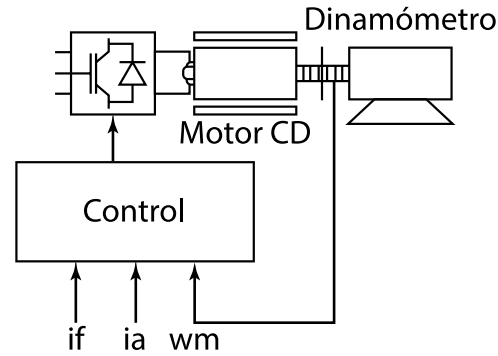

b)

Figura 1. a) Sistema eólico virtual y b) Emulador de turbina de viento.

\section{A. Modelo de la turbina eólica}

Una turbina eólica es una máquina rotatoria cuyo proceso aerodinámico consiste en la generación de energía mecánica a partir de la energía cinética del viento que incide sobre los alabes de la turbina. Típicamente, las turbinas de viento están compuestas por tres alabes montados alrededor de un eje que se conecta a una caja de engranajes, el cual se acopla a un generador eléctrico ${ }^{[1]}$. La eficiencia de la turbina eólica se determina en base al límite de Betz, que es un indicador de la potencia máxima que puede ser extraída en la flecha de la turbina a partir de la energía cinética del viento. La potencia mecánica desarrollada por una turbina eólica se aproxima mediante la siguiente expresión ${ }^{[7]}$ :

$$
P_{m}=\rho_{a}\left(\frac{1}{2} \pi R_{t}^{2}\right) C_{p} v_{w}^{3}
$$

donde $\rho_{\alpha}$ representa la densidad del aire en $\mathrm{kg} / \mathrm{m}^{2} ; R_{t}$ corresponde al radio de la turbina, en $\mathrm{m} ; C_{p}$ representa el coeficiente de potencia; y $v_{w}$ la velocidad del viento que incide en la turbina, en $\mathrm{m} / \mathrm{s}$. Es muy importante resaltar que la potencia desarrollada por la turbina (1) está en función de la velocidad del viento elevada al cubo, y es esta una característica muy relevante y atractiva en la generación de energía eléctrica mediante sistemas eólicos.

El coeficiente de potencia en (1) representa la eficiencia aerodinámica de la turbina, y está dada por la relación entre la energía mecánica extraída por la turbina y la energía cinética del viento que incide en los alabes. Por otro lado, la razón de velocidad de punta $\lambda$ define la relación entre la velocidad tangencial en el extremo de un alabe con la velocidad del viento, y está dada por ${ }^{[7]}$ :

$$
\lambda=\frac{\omega_{t b} R_{t}}{v_{\omega}}
$$

donde $\omega_{t b}$ representa la velocidad angular de la turbina en $\mathrm{rad} / \mathrm{s}, R_{t}$ el radio de la turbina y $\mathrm{v}_{w}$ la velocidad del viento. El coeficiente de potencia varía en función del parámetro $\lambda$ y se puede modelar como se muestra en la siguiente ecuación ${ }^{[6]}$ :

$$
C_{p}(\lambda, \beta)=c_{1}\left(\frac{c_{2}}{\lambda_{i}}-c_{3} \beta-c_{4}\right) e^{\left(-\frac{c_{5}}{\lambda_{i}}\right)}+c_{6} \lambda
$$

donde:

$$
\frac{1}{\lambda_{i}}=\frac{1}{\lambda+0.08 \beta}-\frac{0.035}{\beta^{3}+1}
$$

las constantes del coeficiente de potencia (3) definen las características aerodinámicas del diseño de turbina en particular, y son: $c_{1}=0.5176, c_{2}=116, c_{3}=0.4, c_{4}=$ $5, c_{5}=21, c_{6}=0.0068^{[6]} . \beta$ es el ángulo de paso de los alabes de la turbina eólica y $\beta=0$ al considerar una turbina con alabes fijos, es decir, sin control de la posición del ángulo de paso del alabe.

La representación gráfica del coeficiente de potencia (3) se presenta en la Figura 2, la cual fue realizada en el programa Matlab, en donde el coeficiente de potencia máximo de 0.5263 se presenta en un valor de $\lambda$ de 11.63 . 


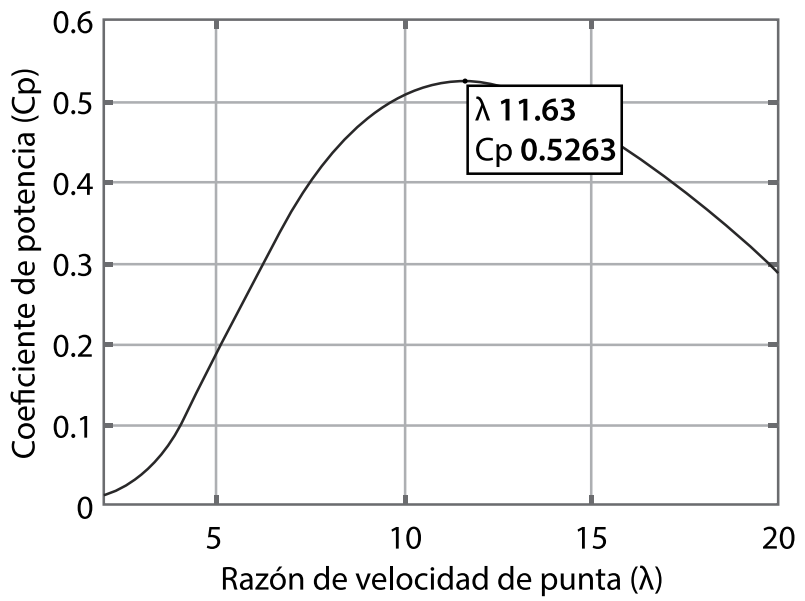

Figura 2. Coeficiente de potencia de la turbina.

El torque mecánico producido por la turbina puede ser aproximado con la siguiente ecuación, la cual resulta de dividir la potencia mecánica desarrollada en la flecha (1) entre la velocidad angular ${ }^{[8]}$ :

$$
T_{t b}=\frac{K_{a} R_{t} C_{p}}{\lambda} v_{w}^{2}
$$

La estrategia para realizar la mímica de la operación de la turbina de viento consiste en la programación virtual en Simulink/Matlab de la ecuación de oscilación mecánica que establece el equilibrio de fuerzas torsionales entre la turbina eólica y el generador y cuya expresión es ${ }^{[9]}$ :

$$
\frac{d \omega_{m}}{d t}=\frac{1}{J_{t}}\left(\frac{T_{t b}}{n_{g b}}-T_{g}-B_{t} \omega_{m}\right)
$$

donde $T_{t b}$ es el torque mecánico desarrollado por la turbina, $T_{g}$ es el par electromagnético producido por el generador de inducción, $J_{t}$ es la suma de los momentos de inercia de la turbina y del generador, $B_{t}$ es la suma de los coeficientes de fricción de la turbina y del generador, y $n_{g b}$ es la relación de dientes de la caja de engranajes que existe entra la turbina y el generador para acoplar la baja velocidad de la turbina con la velocidad alta del generador.

La velocidad angular como variable de salida del exosistema (6) define la velocidad de referencia para el control en lazo cerrado del seguimiento de velocidad del motor de corriente directa. Una vez que el motor siga la velocidad de referencia definida en (6) se cumple el objetivo de emular la operación de la turbina eólica. El exosistema (6) tiene como entrada la velocidad del viento, la cual es simulada mediante un oscilador que va de 4 a $6 \mathrm{~m} / \mathrm{s}$ para establecer un rango de variación en la velocidad de la turbina.

\section{B. Modelo del motor de CD}

El motor de corriente directa es una máquina rotativa que convierte la energía eléctrica de alimentación en energía mecánica en su flecha y se caracteriza por operar en condiciones de par máximo ${ }^{[10,11]}$. El motor de CD es muy versátil y se puede controlar su velocidad en un amplio rango con facilidad, al variar el voltaje de alimentación del devanado de armadura. El modelo matemático del motor de corriente directa con magnetización constante tiene la siguiente representación ${ }^{[9]}$ :

$$
\begin{gathered}
\frac{d \omega_{m}}{d t}=\frac{1}{J_{m}}\left(K_{m} i_{f} i_{a}-B_{m} \omega_{m}-T_{L}\right) \\
\frac{d i_{a}}{d t}=-\frac{1}{L_{a}}\left(R_{a} i_{a}+K_{f} i_{f} \omega_{m}-u_{a}\right)
\end{gathered}
$$

donde $\omega_{m}$ e $i_{a}$ son la velocidad mecánica y la corriente de armadura respectivamente, y son las variables de estado; $i_{f}$ es la corriente de campo que se mantiene constante; $K_{m}$ y $K_{f}$ son constantes del motor con el mismo valor numérico, pero con diferentes unidades; $B_{m}$ es el coeficiente de fricción; $T_{L}$ es el par de carga; $R_{a}$ es la resistencia de armadura; $L_{a}$ es la inductancia de armadura y $u_{a}$ es el voltaje de alimentación al devanado de armadura y es la entrada de control de la planta. El modelo del motor de CD (7) es un sistema lineal de segundo orden con perturbación externa, que representa el par de la carga, y una entrada que se puede controlar $u_{a}$, mediante la técnica de modulación por ancho de pulso, por lo tanto, solo se puede seleccionar una variable de salida a controlar que para nuestro sistema es la velocidad del rotor $\omega_{m}$.

\section{Estimación de parámetros}

Los parámetros eléctricos y mecánicos del motor de corriente directa (LabVolt, modelo 8211), considerando su acoplamiento mecánico con el dinamómetro/ motor de impulsión (LabVolt, modelo 8960) se obtuvieron en forma experimental, utilizando la tarjeta de adquisición de datos dSPACE.

Resistencia de armadura. La resistencia de armadura $R_{a}$ se estimó empleando el método voltímetro/amperímetro, impulsando la máquina de corriente directa totalmente desmagnetizada con un motor de impulsión a la velocidad nominal. Con una fuente de corriente 
directa convencional se desmagnetiza la máquina para que el voltaje inducido en el devanado de armadura sea nulo y se obtienen varios pares ordenados de voltaje y corriente, desmagnetizando la máquina en cada punto y finalizando la prueba en el valor de la corriente nominal de armadura. Posteriormente se realiza un ajuste de los puntos obtenidos a un polinomio lineal mediante la aplicación de mínimos cuadrados y de esta forma se consigue la pendiente que corresponde a la resistencia de armadura y el cruce por el eje $y$, que corresponde a la caída de voltaje en las escobillas.

Inductancia de armadura. La inductancia de armadura $L_{a}$ se estima mediante una prueba dinámica, impulsando a la máquina de corriente directa totalmente desmagnetizada con el motor de impulsión a la velocidad nominal. Una vez girando en estado estable el grupo de máquinas, se aplica un escalón de voltaje con magnitud baja, para provocar un transitorio en la corriente de armadura, el cual se estabiliza en su valor nominal. Del transitorio capturado se estima la constante de tiempo de crecimiento de la corriente y posteriormente se calcula la inductancia de armadura con la siguiente expresión: $L_{a}=\tau_{a} R_{a}$.

Constante del motor. En el proceso de estimación de la constante de motor $K_{f}$, el motor de impulsión se acopló a la máquina de corriente directa operando en modo generador en condiciones de vacío y con un nivel de magnetización definido por el valor de la corriente de campo. El motor de impulsión se hizo girar a diferentes velocidades alrededor de la velocidad nominal y como el generador de corriente directa opera en condiciones de vacío, el voltaje inducido en el devanado de armadura, debido a la magnetización de generador de CD se refleja en sus terminales, por lo que la constante de motor $K_{f}$ se puede determinar mediante la siguiente expresión: $K_{f}=E_{a} / \omega_{m}$, valor que se mantiene constante en las diferentes velocidades de la prueba.

Coeficiente de fricción. El coeficiente de fricción del grupo de máquinas: motor $\mathrm{CD} /$ dinamómetro, se estimó con ambas acopladas mediante una banda dentada en condiciones de par de carga nulo y en estado estable, por lo que el par electromagnético desarrollado por el motor de CD únicamente alimenta las pérdidas por fricción en los rodamientos de las dos y, por lo tanto, a partir de la ecuación de estado mecánica del modelo del motor de CD (7), el coeficiente de fricción se calcula con la siguiente expresión: $B_{m}=T / \omega_{m}$.
Momento de inercia. El momento de inercia $J_{m}$ se estima en forma dinámica a partir de la ecuación de oscilación mecánica del grupo de máquinas: motor $\mathrm{CD} /$ dinamómetro (7). Una vez que el motor opera a velocidad nominal y en condiciones de vacío, se procede a desenergizar el motor y a capturar el proceso transitorio de desaceleración de este. Se linealiza la curva de desaceleración en la vecindad de una velocidad base $\omega_{0}$, y se procede a aplicar la siguiente relación: $J_{m}=-B_{m} \Delta t / \Delta \omega_{0}$.

En la Tabla 1 se presentan los parámetros eléctricos y mecánicos obtenidos en forma experimental.

TABLA 1.

PARÁmetros DEL MOTOR DE CD

\begin{tabular}{|l|c|}
\hline \multicolumn{1}{|c|}{ NomвRE } & VALOR \\
\hline Resistencia de armadura, $R_{a}$ & $6.8 \Omega$ \\
\hline Inductancia de armadura, $L_{a}$ & $75 \mathrm{mH}$ \\
\hline Constante del motor, $K_{f}$ & 2.57 \\
\hline Momento de inercia, $J_{m}$ & $0.0036 \mathrm{~N} \cdot \mathrm{m} \cdot \mathrm{s}^{2}$ \\
\hline Coeficiente de fricción, $B_{m}$ & $0.0022 \mathrm{~N} \cdot \mathrm{m} \cdot \mathrm{s}$ \\
\hline Corriente de campo, $I_{f}$ & $0.25 \mathrm{~A}$ \\
\hline
\end{tabular}

\section{Observador de par}

En el diseño de sistemas de control, algunas variables de estado no son medibles o el costo de los sensores es muy elevado, por lo que es práctica común el diseño de observadores para estimar. En el caso del motor de CD, el modelo del observador de par de la carga es de orden reducido y su representación se basa en el modelo en espacio de estados del motor de CD (7). No obstante, el observador de par incluye un término adicional en cada ecuación de estado que sirve para corregir dinámicamente los errores de observación de la velocidad y del par. Su modelo es el siguiente:

$$
\begin{aligned}
& \frac{d}{d t} \hat{\omega}_{m}=-\frac{1}{\tau_{m}} \hat{\omega}_{m}+\frac{K_{m}}{J_{m}} i_{f} i_{a}-\frac{1}{J_{m}} \hat{T}_{L}+l_{1}\left(\omega_{m}-\hat{\omega}_{m}\right) \\
& \frac{d}{d t} \hat{T}_{L}=l_{2}\left(\omega_{m}-\hat{\omega}_{m}\right)
\end{aligned}
$$

donde $\hat{\omega}_{m}$ es la velocidad mecánica observada, $\tau_{m}$ es la constante de tiempo del motor, $K_{m}$ es la constante de motor, $i_{f}$ es la corriente de campo, $i_{a}$ es la corriente de armadura, $J_{m}$ es el momento de inercia, $\hat{T}_{L}$ es el par de carga, $l_{1}$ y $l_{2}$ son constantes definidas para estabilizar y desvanecer asintóticamente en cero el error de ob- 
servación de velocidad y de par de carga, respectivamente.

Se definen las variables de error de observación para la velocidad mecánica y el par de carga como sigue:

$$
\begin{aligned}
& \tilde{\varepsilon}_{1}=\omega_{m}-\hat{\omega}_{m} \\
& \tilde{\varepsilon}_{2}=T_{L}-\hat{T}_{L}
\end{aligned}
$$

Al calcular las dinámicas del error de observación tanto para la velocidad como para el par de carga, se obtiene el siguiente sistema homogéneo:

$$
\begin{aligned}
& \dot{\varepsilon}_{1}=-\left(\frac{1}{\tau_{m}}+l_{1}\right) \tilde{\varepsilon}_{1}-\frac{1}{J_{m}} \tilde{\varepsilon}_{2} \\
& \dot{\tilde{\varepsilon}}_{2}=-l_{2} \tilde{\varepsilon}_{1}
\end{aligned}
$$

Además, tomando como referencia los valores propios del modelo del motor de CD (7), se establecen los polos deseados de la dinámica del observador y se comparan con las raíces del polinomio característico del sistema con errores de observación (10), obteniéndose los valores de las constantes $l_{1}$ y $l_{2}$ mediante las siguientes relaciones:

$$
\begin{aligned}
& l_{1}=p_{1}+p_{2}-\frac{B_{m}}{J_{m}} \\
& l_{2}=-J_{m} p_{1} p_{2}
\end{aligned}
$$

Los polos deseados del sistema (11) pueden ser del orden de tres veces más rápidos que los polos del modelo del motor en lazo abierto (7).

\section{E. Ley de control}

En el proceso de diseño del sistema de control de velocidad del motor de CD se procede a establecer un modelo dinámicamente equivalente al sistema (7), pero con variables de error de seguimiento. Esta nueva representación del modelo se denomina la forma canónica controlable y es la siguiente:

$$
\begin{aligned}
\dot{\varepsilon}_{1}= & \varepsilon_{2} \\
\dot{\varepsilon}_{2}= & -\left(\frac{K_{f}^{2} i_{f}^{2}}{J_{m} L_{a}}+\frac{1}{\tau_{m} \tau_{a}}\right) \varepsilon_{1}-\left(\frac{1}{\tau_{m}}+\frac{1}{\tau_{a}}\right) \varepsilon_{2}+\ddot{\omega}_{r e f}+\left(\frac{1}{\tau_{m}}+\frac{1}{\tau_{a}}\right) \dot{\omega}_{r e f} \\
& +\left(\frac{K_{f}^{2} i_{f}^{2}}{J_{m} L_{a}}+\frac{1}{\tau_{m} \tau_{a}}\right) \omega_{r e f}+\frac{1}{J_{m}} \dot{T}_{L}+\frac{1}{J_{m} \tau_{a}} T_{L}-\frac{K_{f} i_{f}}{J_{m} L_{a}} u_{a}
\end{aligned}
$$

Esta nueva representación es de segundo orden y para proponer una ley de control robusta de primer orden, es necesario definir un nuevo sistema, como sigue:

$$
s=C_{1} \varepsilon_{1}+\varepsilon_{2}
$$

cuya dinámica es:

$$
\dot{s}=C_{1} \dot{\varepsilon}_{1}+\dot{\varepsilon}_{2}
$$

Si se sustituye (12) en (14), se observa que la entrada de control está presente en la primer derivada del sistema (13) y por lo tanto se puede escoger una técnica de control de primer orden cuyo argumento es la variable $s$. La ley de control que se aplica en el controlador de velocidad es la técnica super-twisting de modos deslizantes de segundo orden, la cual es ${ }^{[11,12]}$ :

$$
\begin{aligned}
& u_{a}(t)=\lambda|s|^{1 / 2} \operatorname{sign}(s)+v \\
& \dot{v}=\alpha \operatorname{sign}(s)
\end{aligned}
$$

La ley de control fuerza a cero la variable $s$ y su primera derivada, con lo que se obtiene un movimiento asintótico del error de seguimiento de velocidad, de la forma:

$$
\dot{\varepsilon}_{1}=-C_{1} \varepsilon_{1}
$$

donde el polo $C_{1}$ debe ser más rápido que los polos del modelo del motor de CD (7), para influir en la rapidez del sistema de control de velocidad en lazo cerrado y se cumpla el objetivo de emular la operación de la turbina al resolver el problema de seguimiento de trayectoria.

\section{RESULTADOS}

Para verificar el desempeño y robustez del emulador de la operación de la turbina eólica, se implementó en tiempo real el controlador de velocidad del motor de CD. El hardware consiste en el kit dSPACE DS1103, que incluye una tarjeta de control y adquisición de datos, un software de visualización y una interfaz de entradas y salidas; un motor de corriente directa acoplado a un dinamómetro con una banda dentada, ambos de la marca LabVolt; y un inversor tipo puente con IGBT LabVolt. Las ganancias de sintonización del controlador super-twisting son $\lambda=0.075$ y $\alpha=4.7$, con un polo en el seguimiento de velocidad de $C_{1}=220$. La estimación del par de la carga se realizó con un observador asintótico cuyas ganancias de sintonización son $l_{1}=450$ y $l_{2}=30$. En las Figuras 3 y 4 se muestra el equipo de laboratorio utilizado para la implementación del emulador de la operación de la turbina eólica en tiempo real. 


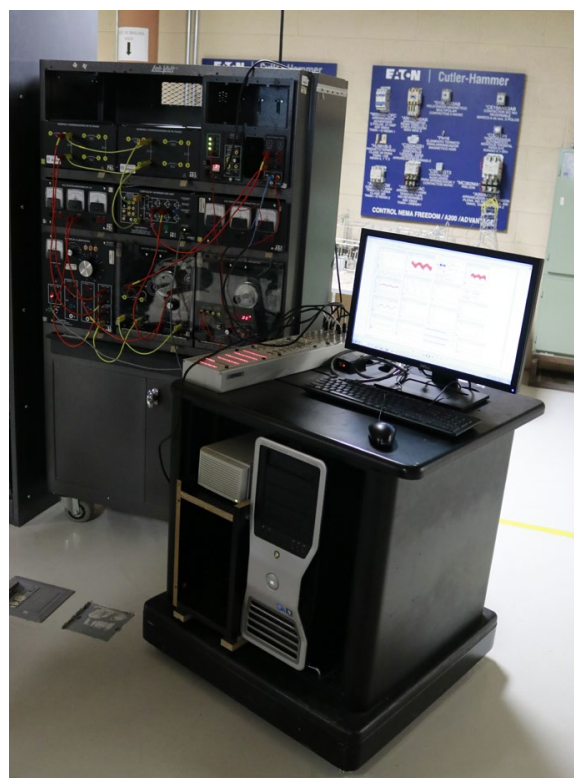

Figura 3. Interfaz de prueba en laboratorio.

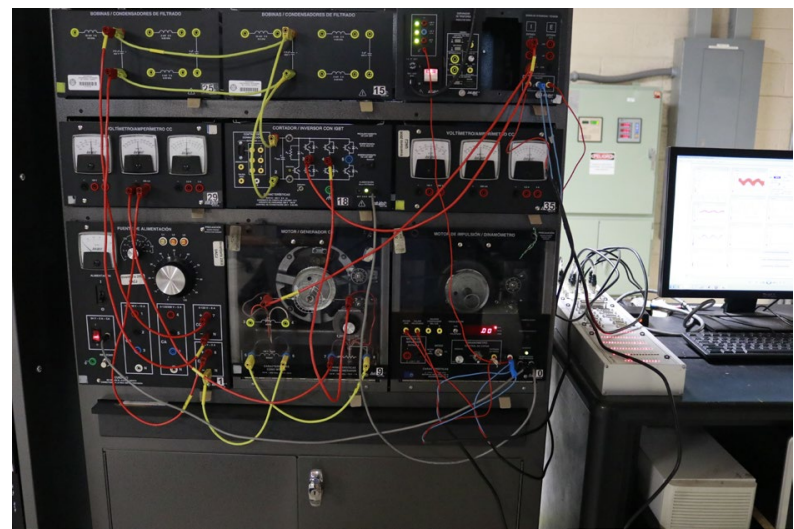

Figura 4. Motor CD - Dinamómetro LabVolt.

En la Figura 5 se muestra el desempeño del emulador de la turbina eólica, en donde la velocidad del motor de CD sigue con buena aproximación la oscilación de velocidad de referencia definida por el exosistema (6) en un rango de 1130 a $1740 \mathrm{rpm}$. En la Figura 6 se muestra el error de velocidad y la entrada de control que utiliza el algoritmo super-twisting, la cual se acondiciona utilizando la técnica de modulación por ancho de pulso con una frecuencia de conmutación de $1 \mathrm{kHz}$. La entrada de control oscila entre 80 y $130 \mathrm{~V}$.

En la Figura 7 se muestran los parámetros de la turbina: el coeficiente de potencia y el parámetro $\lambda$. Es importante resaltar que el coeficiente de potencia se mantiene en un valor constante de 0.5017 , lo cual indica que se está maximizando la captura de energía el viento en la turbina. Por otra parte, en la Figura 8 se muestra la señal de oscilación del viento que varía en un rango de 4 a $6 \mathrm{~m} / \mathrm{s}$, provocando un par en la turbina que oscila entre 0.4 y $1 \mathrm{~N} \cdot \mathrm{m}$. En la Figura 9 se muestran las señales de corriente de armadura y de campo. Por último, en la Figura 10 se expone el desempeño del observador del par de carga, en donde el par del generador eléctrico, como salida del exosistema (6), se alimenta como entrada analógica al dinamómetro y la estimación del par se realiza satisfactoriamente con el observador del par de carga del motor de CD.

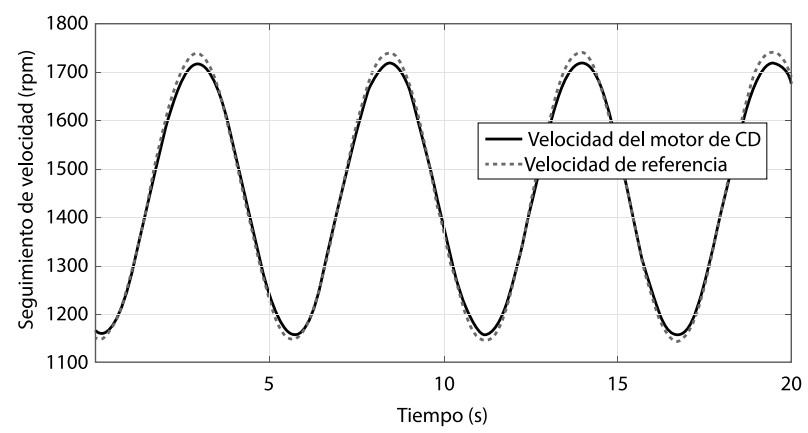

Figura 5. Desempeño del emulador de turbina.

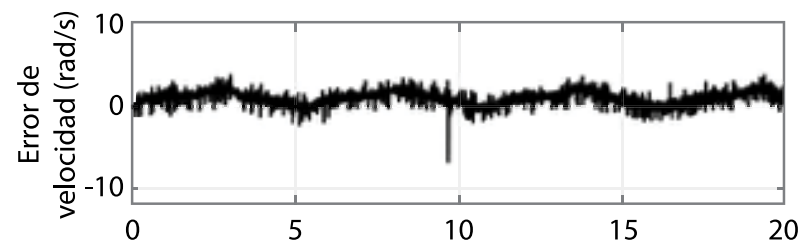

a) Tiempo (s)

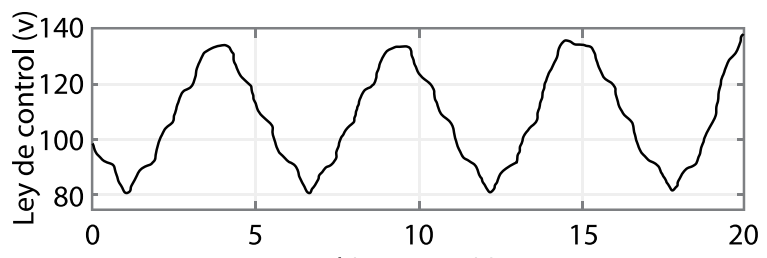

b) Tiempo (s)

Figura 6. a) Señal de superficie y b) ley de control.
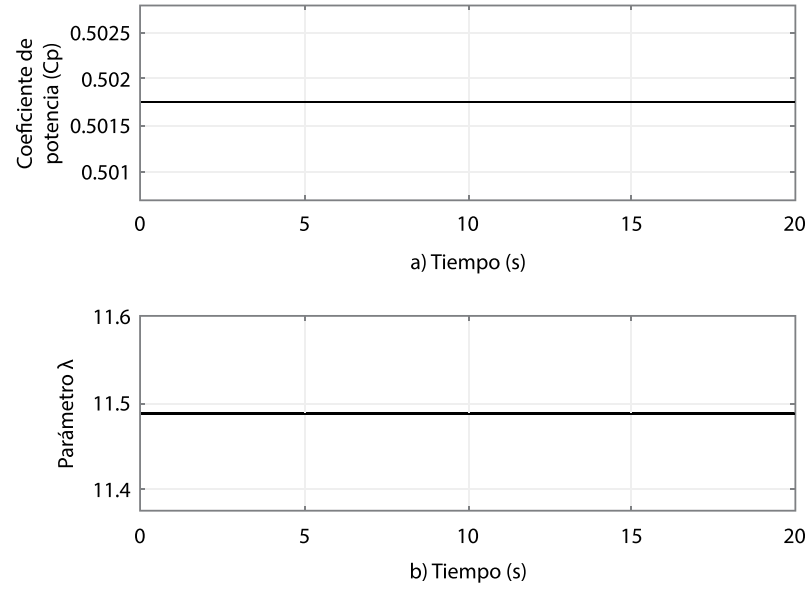

Figura 7. a) Coeficiente de potencia y b) parámetro $\lambda$. 

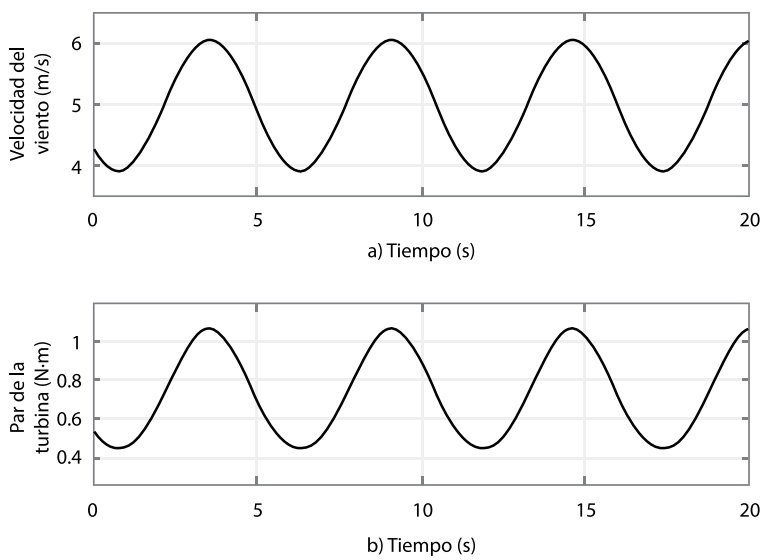

Figura 8. a) Velocidad del viento y b) par de la turbina.
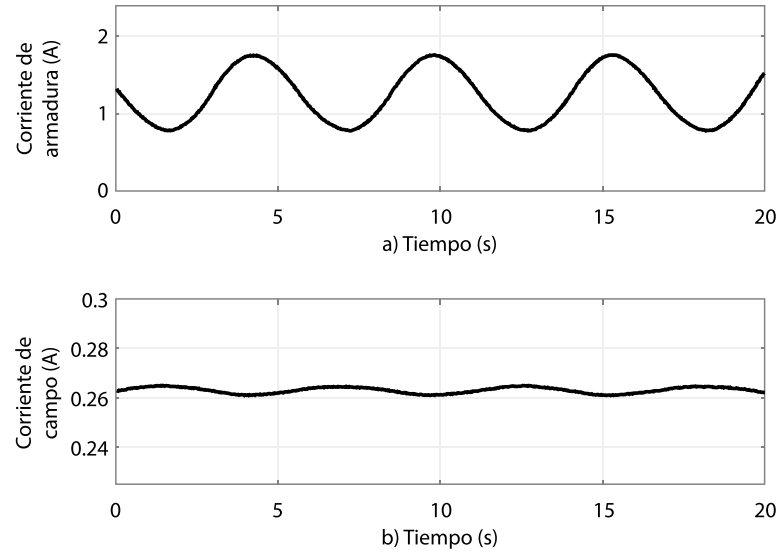

Figura 9. a) Corriente de armadura y b) corriente de campo.

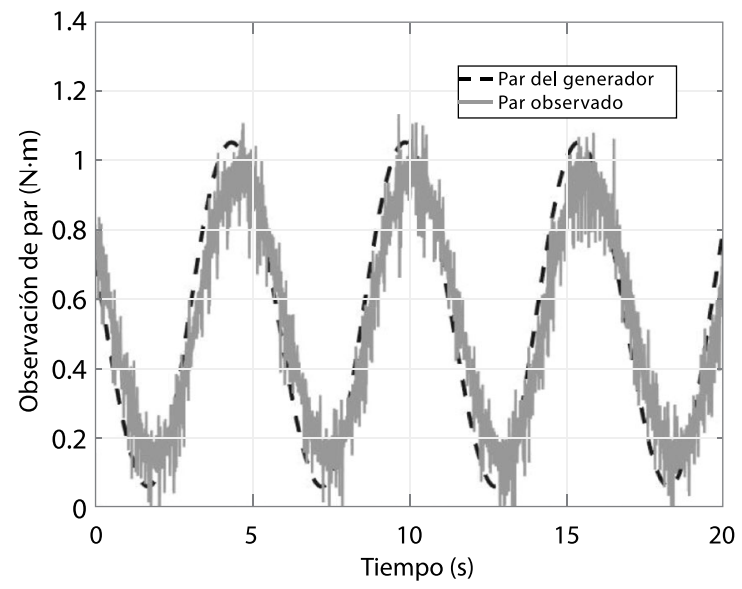

Figura 10. Observación de par de carga.

\section{CONCLUSIONES}

En el presente artículo se ha establecido una metodología para el diseño e implementación en tiempo real del emulador de turbina eólica, el cual funciona cuando se realiza el seguimiento de trayectoria del controlador de velocidad del motor de $\mathrm{CD}$, teniendo como referencia la velocidad de salida de la flecha del sistema virtual turbina-generador, que tiene como entrada un perfil de viento virtual.

Se aplica la ley de control super-twisting de modos deslizantes de segundo orden en el diseño del controlador, la cual se distingue por ser muy robusta ante la presencia de perturbaciones externas al sistema y a la variación de parámetros. Con la ley de control se fuerza la variable de superficie (13) a cero en tiempo finito, con lo que se consigue un movimiento asintótico hacia cero del error de seguimiento de velocidad, logrando con esto el objetivo de control, que consiste en emular la operación de la turbina eólica.

El coeficiente de potencia (3) y la razón de velocidad de punta de la turbina (2) se mantienen en un valor constante, lo que indica que se realiza la captura máxima de energía de viento. Los resultados experimentales de la operación del emulador de la turbina eólica, muestran robustez del controlador, en donde el seguimiento de trayectoria de la velocidad de la turbina se realiza en forma satisfactoria mediante el control de velocidad del motor de CD.

Una vez operando satisfactoriamente el emulador de la turbina, no es necesario contar con la infraestructura de la turbina eólica y el túnel de viento y pueden enfocarse proyectos en el diseño de sistemas de control en lazo cerrado aplicados al generador eléctrico y a la interconexión de la energía eléctrica generada con la red eléctrica.

\section{REFERENCIAS}

[1] M. Hasan Ali, Wind Energy Systems: Solutions for Power Quality and Stabilization. Nueva York: CRC Press, 2017.

[2] L. Bennaouinate, M. Khafallah, A. Mesbahi y A. Martinez, "Development of a useful Wind Turbine Emulator Based on Permanent Magnet DC Motor", en 14th International Multi-Conference on Systems, Signals \& Devices (SSD), Marruecos, marzo 28-31, 2017. pp. 44-48. doi: 10.1109/SSD.2017.8166972.

[3] J. M. Guerrero, C. Lumbreras, D. Diaz Reigosa, P. Garcia y F. Briz, "Control and Emulation of Small Wind 
Turbines Using Torque Estimators", IEEE Transactions on industry applications, vol. 53, no. 5, pp. 4863-4876, 2017. doi: 10.1109/TIA.2017.2708027.

[4] Y. Sirouni, S. El Hani, N. Naseri, A. Aghmadi y K. El Harouri, "Design and Control of a Small Scale Wind Turbine Emulator with a DC Motor", 2018 6th International Renewable and Sustainable Energy Conference (IRSEC 2018), Marruecos, diciembre 5-9, 2018. pp. 547 552. doi: 10.1109/IRSEC.2018.8702899.

[5] O. A. Morfin, R. Ruiz-Cruz, F. Ornelas-Tellez, A. Campos-Valdillez, R. Ramírez-Betancour, N. Padilla y J. Gándara, "Maximum wind energy capture in a wind system using a doubly-fed induction generator", 2018 IEEE International Autumn Meeting on Power, Electronics and Computing (ROPEC), Ixtapa, noviembre 14-16, 2018. pp. 1-6. doi: 10.1109/ROPEC.2018.8661374

[6] H. Voltolini, M. H. Granza, J. Ivanqui y R. Carlson, "Modeling and simulation of the Wind Turbine Emulator using induction motor driven by torque control inverter", 2012 10th IEEE/IAS International Conference on Industry Applications, Fortaleza, noviembre 5-7, 2012. pp. 1-6. doi: 10.1109/INDUSCON.2012.6453399
[7] T. Burton, N. Sharpe, N. Jenkis y E. Bossanyi, Wind Energy Handbook. Chichester, U. K.: John Wiley \& Sons, Ltd, 2001.

[8] S. Kouadria, S. Belfedhal, E. Berkouk y Y. Meslem, "Development of real time wind turbine emulator based on DC motor controlled by PI regulator", 2013 Eighth International Conference and Exhibition on Ecological Vehicles and Renewable Energies (EVER), Monte Carlo, marzo 27-30, 2013. p. 5. doi: 10.1109/ EVER.2013.6521602

[9] P. Krause, O. Wasynczuk, S. D. Sudhoff y S. Pekarek, eds., Analysis of Electric Machinery and Drive Systems. USA: IEEE, 2013.

[10] P. C. Sen, Principles of Electric Machines and Power Electronics. USA: Wiley, 2013.

[11] L. Fridman y A. Levant, "Higher Order Sliding Modes", en Sliding Mode Control in Engineering, J. P. Barbot y W. Perruguetti, eds. USA: Marcel Dekker, 2002.

[12] V. Utkin, J. Guldner y M. Shijun, Sliding Mode Control in Electromechanical Systems. Londres: Taylor \& Francis Ltd, 1999. 